\title{
Neurodevelopment of Amazonian Infants: Antenatal and Postnatal Exposure to Methyl- and Ethylmercury
}

\author{
José G. Dórea, ${ }^{1}$ Rejane C. Marques, ${ }^{2}$ and Cintya Isejima ${ }^{3}$ \\ ${ }^{1}$ Department of Nutrition, University of Brasília, 70919-970 Brasília, DF, Brazil \\ ${ }^{2}$ Federal University of Rio de Janeiro, Campus Macaé, 27971-550 Rio de Janeiro, RJ, Brazil \\ ${ }^{3}$ Departament of Statistics, Federal University of São Carlos, 13566-590 São Carlos, SP, Brazil
}

Correspondence should be addressed to José G. Dórea, dorea@rudah.com.br

Received 5 December 2011; Accepted 4 February 2012

Academic Editor: João B.T. Rocha

Copyright () 2012 José G. Dórea et al. This is an open access article distributed under the Creative Commons Attribution License, which permits unrestricted use, distribution, and reproduction in any medium, provided the original work is properly cited.

\begin{abstract}
Neurodevelopment as Gesell development scores (GDSs) in relation to mercury exposure in infants ( $<6$ months of age) of one urban center and two rural villages, respectively, of fisherman and cassiterite miners. Mean total hair- $\mathrm{Hg}(\mathrm{HHg})$ concentrations of infants from Itapuã $(3.95 \pm 1.8 \mathrm{ppm})$ were statistically $(P=0.0001)$ different from those of infants from Porto Velho $(3.84 \pm$ $5.5 \mathrm{ppm})$ and Bom Futuro $(1.85 \pm 0.9 \mathrm{ppm})$. Differences in vaccine coverage among these populations resulted in significantly higher $(P=0.0001)$ mean ethylmercury $(\mathrm{EtHg})$ exposure in urban infants $(150 \mu \mathrm{g})$ than in infants from either village $(41.67 \mu \mathrm{g}$, Itapuã; $42.39 \mu \mathrm{g}$, Bom Futuro). There was an inverse significant (Spearman $r=-0.2300 ; P=0.0376$ ) correlation between $\mathrm{HHg}$ and GDS for infants from Porto Velho, but not for the rural infants from Bom Futuro (Spearman $r=0.1336 ; P=0.0862$ ) and Itapuã (Spearman $r=0.1666 ; P=0.5182$ ). Logistic regression applied to variables above or below the median GDS showed that EtHg exposure (estimated probability $=-0.0157 ; P=0.0070$ ) and breastfeeding score (estimated probability $=-0.0066$; $P=0.0536$ ) score were significantly associated with GDS. Conclusion. In nurslings whose mothers are exposed to different levels of fish-MeHg (HHg), a higher score of neurological development at six months was negatively associated with exposure to additional TCV-EtHg. Results should be interpreted with caution because of unaccounted variables.
\end{abstract}

\section{Introduction}

The first years are critical for children's future health and development. A healthy infanthood can be monitored by physical growth and neurodevelopment (social, emotional, language, and cognitive domains). There are sufficient studies documenting the vulnerability of the developing central nervous system (CNS) to adverse effects of neurotoxicants [1], and mercury is the most prevalent of these among fisheating populations. Among high consumers of seafood it has been shown that maternal exposure to methylmercury $(\mathrm{MeHg})$ during pregnancy can affect neurodevelopment of children [2]. Indeed, Björnberg et al. [3] have shown that infant exposure to mercury is higher before birth than during the breastfeeding period.

Nutrition, toxic, and stimulatory events in this early phase can influence neurobehavioral scores in childhood $[4,5]$ by interfering with the neural programming of the
CNS. During critical periods of early CNS development, depending on the neurotoxic insult, neurological delays can have an indelible effect. Indeed, the developmental origin of neurological diseases/dysfunction is a concept supported by current epidemiological studies [1]. However, it is recognized that, in most circumstances, exposure occurs to more than one neurotoxic substance. In this regard, Stewart et al. [6] have shown negative association between prenatal $\mathrm{MeHg}$ exposure and neurocognitive tests at 38 months to be higher in preschoolers with higher levels of other neurotoxic substances.

The traditional Amazon lifestyle has been characterized by consumption of abundant fish during pregnancy and breastfeeding [7]. However, the growing human occupation and development of the Western Amazon has brought deforestation by fire (for agriculture) and open-cast mining, both of which cause environmental degradation and pollution. Additionally hydroelectric reservoirs have displaced 
traditional living [8]. It is also amply recognized that in mining areas there are elevated risks of coexposure to neurotoxic elements resulting from ore extraction and metal processing activities [9]. In new agricultural frontiers of the Amazon there is evidence that anthropogenic activities have contributed to the release of naturally accumulated $\mathrm{Hg}$ [10] and have also increased organochlorine residues in the aquatic ecosystem. Rabitto et al. [11] found that fish from the Samuel reservoir are contaminated with DDT residues and also with high $\mathrm{Hg}$ concentrations.

For mothers eating fish there is attendant exposure to $\mathrm{MeHg}[8,9,12]$. However, not only do other neurotoxicants reach the Amazonian aquatic food web, but there is also iatrogenic exposure to ethylmercury (EtHg) in thimerosalcontaining vaccines (TCVs) which are the norm in Brazil's successful immunization program [13]. Despite early-life exposures to preservative (thimerosal) and adjuvant (aluminum) in TCV given to pregnant mothers and babies [14, 15], studies assessing the neurological status of Amazonian children have only considered fish-MeHg [16], missing differences in TCV-EtHg exposure [17].

In this transitioning demography of the Western Amazon we have shown different levels of environmental $\mathrm{Hg}$ exposure attendant on variations in maternal fish consumption in children. In these studies we addressed preschool (under five years of age) children's growth and neurodevelopment for urban children [13] and for the families of fishermen [8] and tin miners [9]. In the present work we studied environmental differences (due to maternal fish-MeHg) coupled with variations in postnatal TCV coverage between urban and rural infants. In order to capture early effects of pre- and postnatal $\mathrm{Hg}$ exposure, we compared only six-month-old infants' neurodevelopment in three distinct sociocultural situations in urban (Porto Velho) and rural (fishing village and mining settlement) environments.

\section{Methods}

2.1. Background. We have been studying nutritional status, growth, and neurodevelopment, as well as health of Amazonian children since the late 1990s. In the state of Rondania we have been able to address growth and development in the city of Porto Velho and in impacted rural areas. Results of a longitudinal cohort of 5 years in urban children have appeared in several publications [13]. A second (crosssectional) study started in 2007, aimed at all children under 5 years of age in rural communities formed after displacement of traditional families by the flooding of a hydroelectric reservoir (Itapuã) and new settlers (Bom Futuro) attracted by cassiterite mining $[8,9]$. The protocol of these studies followed ethical recommendations and received approval from the Ethics Committee for Studies in Humans of the Universidade Federal de Rondania [8, 9, 12]; these publications carry maps showing the geographic location of the city of Porto Velho (capital of the state of Rondania) and the studied communities. Written consent was always obtained from the volunteering mother with assured confidentiality. Mothers could withdraw from the study at any time.
2.2. Exposure Assessment and Neurological Tests. The data presented in this paper comprise a specific subset of children 1 to 6 months of age (281) representing all infants surveyed in the two rural communities of Itapuã $(n=33$; former subsistence fisherman families) and Bom Futuro ( $n=166$; families of cassiterite mining settlers) and the first part of the study with a sample of urban infants of Porto Velho $(n=82)$; respective publications on linear growth and neurodevelopment for preschool children (1 to 60 months of age) have appeared elsewhere $[8,9,12]$. We made anthropometric measurements and collected hair for all infants.

Briefly, during home visits we also applied a questionnaire to the mothers to collect information on breastfeeding duration and immunization records. Because of easy access to health facilities the children of Porto Velho received the full immunization scheme. As for the rural villagers, vaccination coverage experiences a number of difficulties and is not always followed as recommended; however, all vaccines applied had been recorded and this information was acquired from the vaccination cards. The total amount of EtHg was computed from information on the TCV (hepatitis $\mathrm{B}$ and DTP) given during the first six months; because all these TCVs are formulated to contain aluminum salts as adjuvant, total amount of adjuvant-Al was also estimated for the vaccinated infant.

During visits, trained professionals conducted interviews and applied the Gesell neurodevelopment tests. The neurodevelopment of infants was assessed by trained professionals using the Gesell Developmental Schedules (GDSs) [18, 19]. The GDSs include reflexes and reactions (voluntary, spontaneous, or learned) as well as postural reactions, hand pressure, locomotion and coordination, constructive ability (which is influenced by motor development), and visible and audible communication; individual reactions regarding people and stimulations (depending mainly on the temperament of the child and the surroundings) were also evaluated [12]. The results were expressed as scores for the assessed domains (motor skills, language development, adaptive behavior, and personal social behaviors).

Breastfeeding for the infants of Porto Velho lasted for six months or more [13], whereas for the rural infants we estimated a breastfeeding score based on the information given at the time of the GDS testing. A score was calculated in relation to the day of the visit and age of the infant (breastfeeding score $=$ age of infant in days/breastfeeding days $\times 100$ ). The published parent publications $[8,9$, 12] describe the protocol and methods for anthropometry and hair-Hg determinations. Strands of hair were collected from the children and were stored, processed, and analyzed according to our routine laboratory protocol after washing with detergents and digesting with acid $[8,9,12]$.

2.3. Statistical Analysis. Data summarization (means, standard deviation) and correlation analysis were done by the statistical packages; Prism software (Prism, version 10IC; GraphPad Software Inc., San Diego, CA, USA) was used to generate graphs and determine Spearman's $(P)$ correlation 
TABLE 1: General characteristics of families and respective infants in the study.

\begin{tabular}{|c|c|c|c|c|}
\hline \multirow[b]{2}{*}{ Communities } & \multicolumn{3}{|c|}{ Mean (SD) } & \multirow{2}{*}{$P$} \\
\hline & Itapuã & Bom Futuro & Porto Velho & \\
\hline \multicolumn{5}{|l|}{ Family } \\
\hline Fish meal, week ${ }^{\mathrm{a}}$ & $2.63(2.70)$ & $2.4(1.4)$ & $1.97(2.8)$ & $<0.000$ \\
\hline Family income US\$ & $227.93(193.9)$ & $225.2(146.1)$ & $247.30(301.8)$ & 0.4771 \\
\hline Maternal education, y & $6.56(3.49)$ & $6(2.7)$ & $8.7(3.5)$ & $<0.000$ \\
\hline \multicolumn{5}{|l|}{ Children } \\
\hline$n$ & 33 & 166 & 82 & \\
\hline \multicolumn{5}{|l|}{ Birth } \\
\hline Weight (g) & $3445.0(510.9)$ & $3278.5(462.8)$ & $3233.2(421.6)$ & 0.0787 \\
\hline Length $(\mathrm{cm})$ & $50.7(2.7)$ & $50.8(2.6)$ & $49.8(2.4)$ & $<0.000$ \\
\hline Age at visit, months & $3.7(1.7)$ & $2.8(1.5)$ & 6 & N.T. ${ }^{c}$ \\
\hline Breastfeeding score $\%^{\mathrm{b}}$ & $96.1(16.9)$ & $81.0(32.6)$ & 100 & $<0.000$ \\
\hline W/H Z-scores & $0.16(1.3)$ & $0.03(1.4)$ & $-1.22(1.2)$ & $<0.000$ \\
\hline H/A Z-scores & $-0.27(1.5)$ & $0.52(0.8)$ & $0.38(1.3)$ & 0.001 \\
\hline W/A Z-scores & $-0.22(1.2)$ & $0.22(0.9)$ & $-0.77(0.7)$ & $<0.000$ \\
\hline TCV-Hg, $\mu \mathrm{g}$ & $41.7(29.8)$ & $42.4(24.5)$ & 150 & $<0.000$ \\
\hline Infant's hair $\mathrm{Hg}, \mu \mathrm{g} \cdot \mathrm{g}^{-1}$ & $3.95(1.8)$ & $1.85(0.9)$ & $3.84(5.5)$ & $<0.000$ \\
\hline
\end{tabular}

${ }^{\mathrm{a}}$ Number of fish meals a week; ${ }^{\mathrm{b}}$ breastfeeding score $=$ age of infant in days/breastfeeding days $\times 100$; ${ }^{\mathrm{c} N}$.T., not tested per se but as age-dependent variable for anthropometry and breastfeeding scores; W/H: weight for height; H/A: height for age, W/A: weight for age; TCV: uptake of mercury in thimerosal-containing vaccines.

between the variables of interest. A logistic regression analysis was used to test the effect of chosen variables on children's GDS by SAS (SAS Institute, Cary, NC, USA) statistical package.

2.4. Controlling for Confounders. Potential confounders known to interfere with neurodevelopment that were part of the study were considered in the logistic regression model. The rationale behind the choice of covariate variables was based on published literature that includes our own work. The frequency of family fish consumption is associated with total mercury concentrations in infants' hair $[20,21]$. Breastfeeding is known to modulate neurobehavior [22] and also to counteract negative effects of maternal exposure to neurotoxic substances [23]. TCV is the only source of EtHg which is a relevant neurotoxic per se and, in the case of vaccines, because of its obligatory association with adjuvant-Al salts [15], it actually represents a combination of thimerosal and aluminum salts sufficiently defined (and constant) for it to be treated as a unit here.

\section{Results}

3.1. General Characteristics and Mercury Exposure. The general characteristics of mothers and infants in the three settings are summarized in Table 1; for all parameters, except family income, there were statistically significant differences. Only breastfeeding score and EtHg exposure showed a similar pattern distinguishing urban from rural settings. In the urban sample, the infants had a full TCV schedule and breastfed for at least six months. Infants' exposure to mercury from maternal fish consumption is represented as $\mathrm{HHg}$ while iatrogenic sources such as EtHg from TCV were estimated from vaccination cards and represent a combination of EtHg and attendant adjuvant-Al. As a result, a more precise (in time, quantity, and route) measure of exposure to EtHg was assessed from vaccine cards, showing a statistically significant difference between groups (Table 1). While infants from Porto Velho had received the full vaccine schedule $(150 \mu \mathrm{g}$ of EtHg), rural infants were less exposed (by approximately one-third) to this mercury source because of age variation, less effective vaccine coverage, and schedule adherence; the overall exposure to EtHg in the rural settings was $42.4 \mu \mathrm{g}$ and $41.7 \mu \mathrm{g}$ of EtHg for Bom Futuro and Itapuã villages, respectively. For statistical modeling and interpretation of the GDS responses, the EtHg exposure actually represents a combination of two neurotoxicants (thimerosal and adjuvant-Al salts); they are an integral part of the vaccine formulation.

Except for the children of Porto Velho who were exclusively breastfed for at least six months (breastfeeding score of $100 \%$ ), breastfeeding for the other groups (Itapuã and Bom Futuro) showed lower scores (Table 1). In order to adjust for these differences at the time of the neurobehavioral testing, this variable is presented as "breastfeeding score" (estimated at the day of the visit). At six months, when the Porto Velho infants were tested, all of them (100\%) were still breastfeeding, whereas the mean lactation score was relatively less (96.0, Itapuã; 81.0, Bom Futuro) for rural infants (Table 1).

In Figure 1 the cumulative distribution of $\mathrm{HHg}$ indicated different patterns of maternal fish consumption in the three settings. The HHg concentrations of the Porto Velho infants showed the highest proportion of low values in sharp contrast with fisherman infants (Itapuã) who showed 


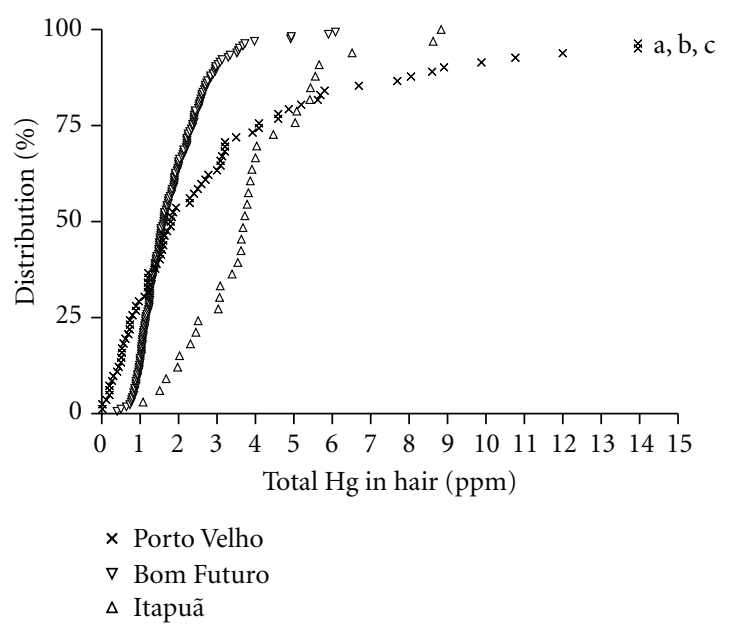

Figure 1: Cumulative percent distribution of total hair $\mathrm{Hg}$ concentrations in infants from different localities and maternal fisheating habits; a, b, c are off-chart values of Porto Velho infants' HHg concentrations, respectively, of 18.3, 26.8, $32.9 \mathrm{ppm}$.

the highest proportion of high values. In fact, the median $\mathrm{HHg}$ of fisherman infants (3.7 ppm) was more than twice the median found in urban infants from Porto Velho (1.8 ppm) and that of the cassiterite mining settlements (1.6 ppm). At this early age, infants' $\mathrm{HHg}$ reflects in utero exposure as well as maternal milk (mostly breastfeeding) exposure; both types of exposure are influenced by maternal fish-eating habits. Indeed, correlation between infants' $\mathrm{HHg}$ and family fish consumption (Figure 2) was statistically significant for each of the three settings: Porto Velho (Spearman $r=0.489, P=$ 0.0001), Bom Futuro (Spearman $r=0.1531, P=0.0489$ ), and Itapuã (Spearman $r=0.3763, P=0.0309$ ).

3.2. Neurodevelopment Scores. The cumulative distribution of the neurodevelopment scores (GDS) is illustrated in Figure 3. A greater proportion of low GDS occurred in infants from Porto Velho, in contrast with a greater proportion of higher scores that occurred in infants from the fishing villages-Itapuã families (Figure 3). It is important to notice that proportionately more infants (95\%) from the Itapuã families showed $\mathrm{HHg}$ above $1.5 \mathrm{ppm}$; in this regard there were far more infants from Bom Futuro (35\%) and Porto Velho (40\%) with $\mathrm{HHg}$ concentrations lower than $1.5 \mathrm{ppm}$ (Figure 1). The box plot in Figure 4 illustrates the general GDS and specific domains tested; collectively, the infants of Itapuã seemed to have a more homogenous neurodevelopment than infants from the other study sites. Thus the pattern of distribution of GDS shows an opposite trend compared with the HHg distribution (Figures 1 and 3).

Because of the differences in mercury exposure (maternal fish consumption and TCV-EtHg) and GDS we tested for correlation between these variables in the three settings. The Spearman correlation analyses of $\mathrm{HHg}$ and GDS are illustrated in Figure 5. While correlation of $\mathrm{HHg}$ and GDS in infants from Porto Velho (Spearman $r=-0.2300$;
$P=0.0376)$ was statistically significant, in infants from Bom Futuro (Spearman $r=0.1336 ; P=0.0862$ ) and in infants from Itapuã (Spearman $r=0.1666 ; P=0.5182$ ) these correlations did not reach statistical significance.

A multivariate model (logistic regression) tested the probability of a child showing a GDS above the median, and results are summarized in Table 2. Among the variables tested, exposure to EtHg and breastfeeding score showed negative associations that were statistically significant (EtHg exposure: estimate $=-0.0157, P=0.0070$; "breastfeeding score": estimate $=-0.0065, P=0.0536)$.

\section{Discussion}

This paper shows differences in pre- and postnatal exposure to organic mercury, revealing negative associations with neurodevelopment in six-month-old infants. Overall, most infants had $\mathrm{HHg}$ above $1.5 \mathrm{ppm}$, but the group from Porto Velho with the lowest median $\mathrm{HHg}$ concentration had a higher frequency of low GDS. Curiously, these infants had the highest exposure to vaccine-EtHg. Although the measured delays were overcome by 36 months [13], these findings are nevertheless important to show that researchers should consider all sources of $\mathrm{Hg}$ in populations using TCVs. Indeed, when vaccine-EtHg exposures are included in statistical model measuring neurological outcome of children there are indications concurring with our results. In South Korea, Lee and Ha [24] observed a marginal level of significance in mental development index related to maternal smoking when considering vaccination history. Also in Poland, Jedrychowski [25] informs that TCV effects on psychomotor development appeared to be significant, although very weak.

Chronic neurotoxic sequelae attributed only to the high burden of $\mathrm{MeHg}$ resulting from subsistence fish eating in the Amazon were found by some but not by others. Neurobehavioral studies in Amazonian children of high fish consumers (mean $\mathrm{HHg}$ concentration $>10 \mathrm{ppm}$ ) tended to show a significant decrease in tested neurobehavioral functions [26, 27], while in other studies (with mean $\mathrm{HHg}$ $<5 \mathrm{ppm}$ ) children showed no such significant effects [28]. Although differences in $\mathrm{Hg}$ exposure may seem to explain differences in study results, the neurodevelopment status of rural Amazonian children is not an exclusive function of the assessed fish-Hg exposure. Fonseca et al. [29] showed that low results in neurofunctional testing among children from isolated communities are related to causes other than $\mathrm{MeHg}$ derived from fish consumption; subsistence riverine children with hair-Hg concentrations 66 times higher than those found in non-Amazonian agrarian communities had comparably poor neurofunctional outcomes.

Grandjean et al. [2] noticed that prenatal low levels of $\mathrm{MeHg}$ exposure that may affect brain function can only be detected after the child is old enough to be tested in cognitive domains. However, addressing neurological development as a function of breastfeeding, Grandjean et al. [30] reported that infants with higher HHg at 12 months of age reached the milestone criteria earlier and attributed this to breastfeeding 


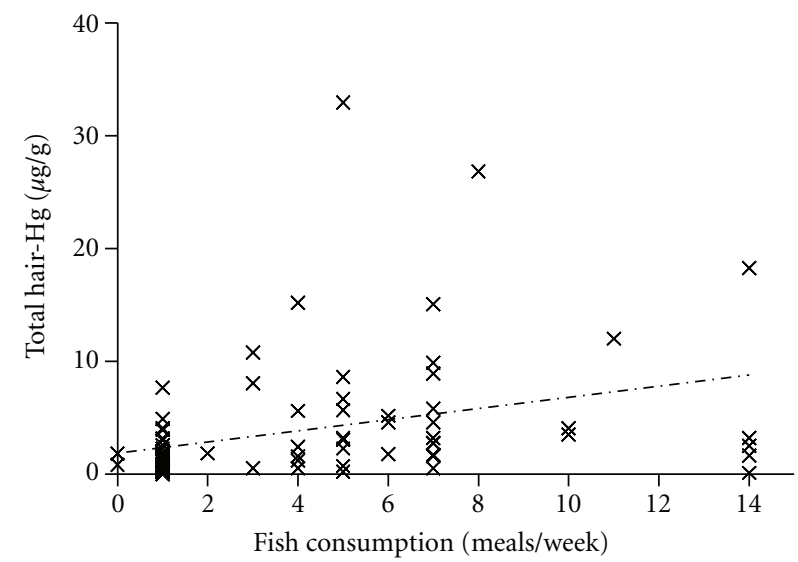

$\times$ Porto Velho

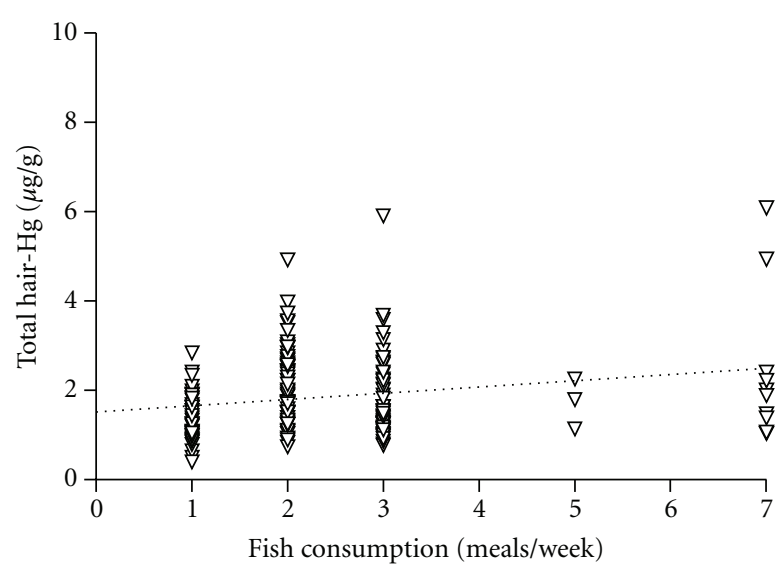

$\nabla$ Bom Futuro

(a)

(b)

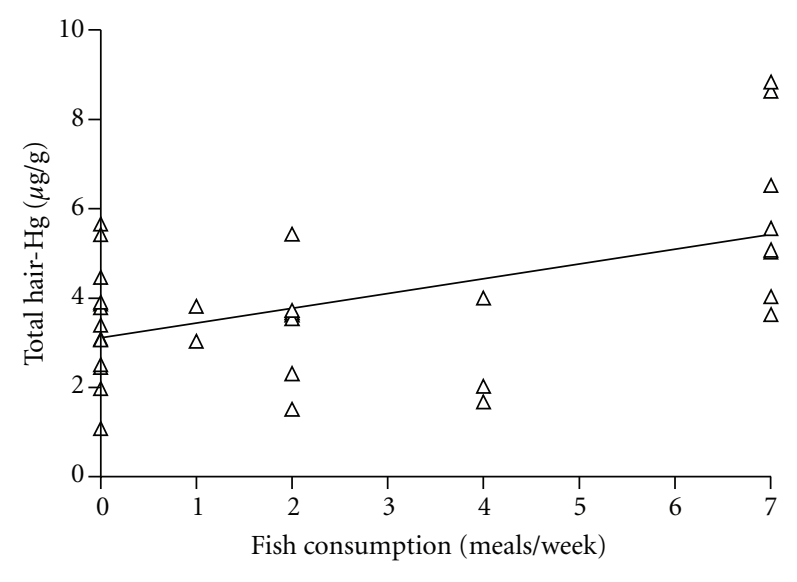

$\Delta$ Itapuã

(c)

FIGURE 2: Scatter plots of infant's total hair-Hg concentrations as a function of maternal fish consumption; the Porto Velho data "high" refers to eating $>2$ fish meals a week.

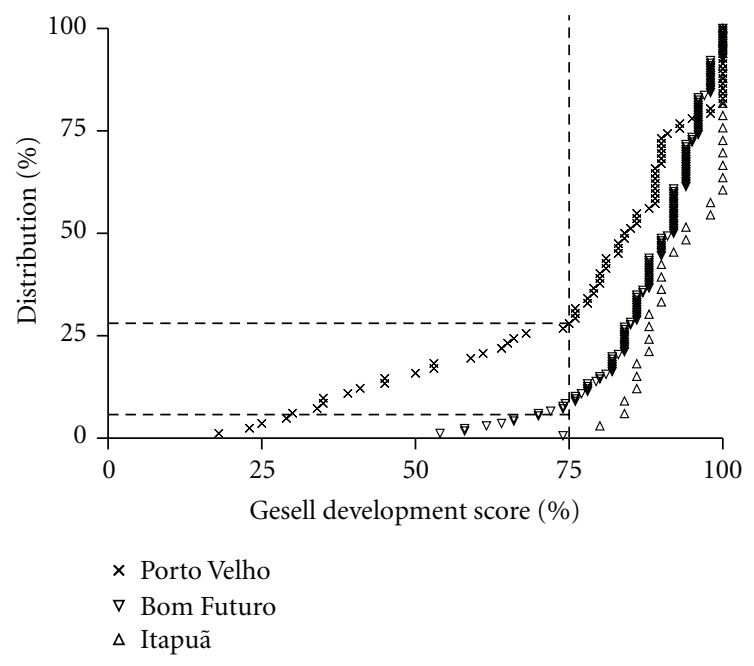

FIGURE 3: Cumulative percent distribution of infants' neurological test at 6 months; Gesell total development quotient. duration. In the infants of Porto Velho neurodevelopmental delays (attributed to Hg that included TCV) were inversely correlated with length of breastfeeding and were overcome by age of 5 yrs [13]. At six months, however, comparing groups of infants with different $\mathrm{Hg}$ exposure (chemical forms and time) it was possible to reveal significant associations with neurobehavioral testing. In this logistic regression breastfeeding seemed less than that associated with TCVEtHg (Table 2).

There are few publications reporting $\mathrm{HHg}$ concentrations in infants who are still under the influence of maternal $\mathrm{Hg}$ exposure. As an exposure marker, $\mathrm{HHg}$ has been reported for babies in Italy [31], in Surinam [32] and in the Faroe Islands [20]. In Italy mean $\mathrm{HHg}$ was $1.22 \mathrm{ppm}$, while in Surinam there was a median of $1.6 \mathrm{ppm}$, and in the Faroe Islands the interquartile range was from 0.46 to $2.04 \mathrm{ppm}$. Babies in the present study showed higher HHg concentrations than in other countries but lower than previously ( $>15$ yrs ago) found in traditional riverine communities of the Rio Madeira 


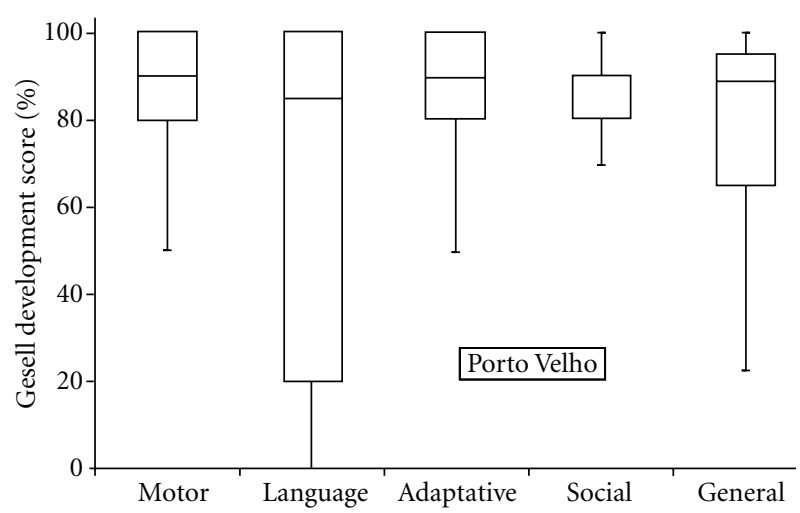

(a)

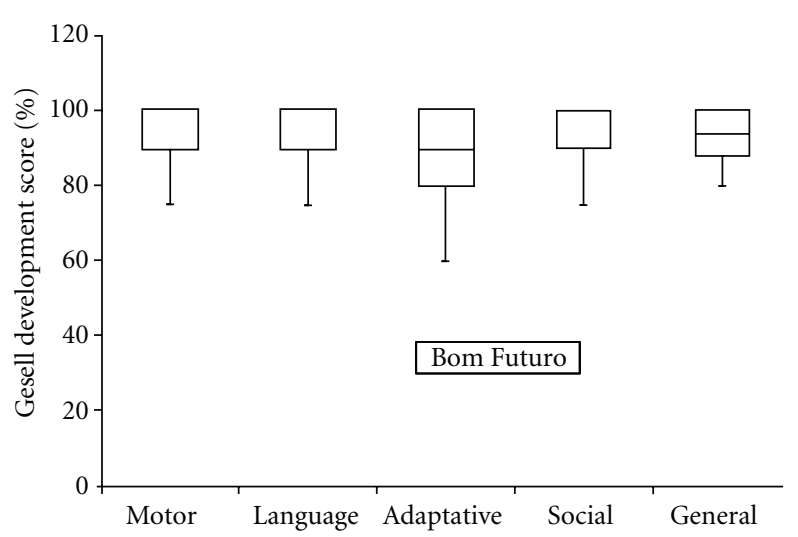

(b)

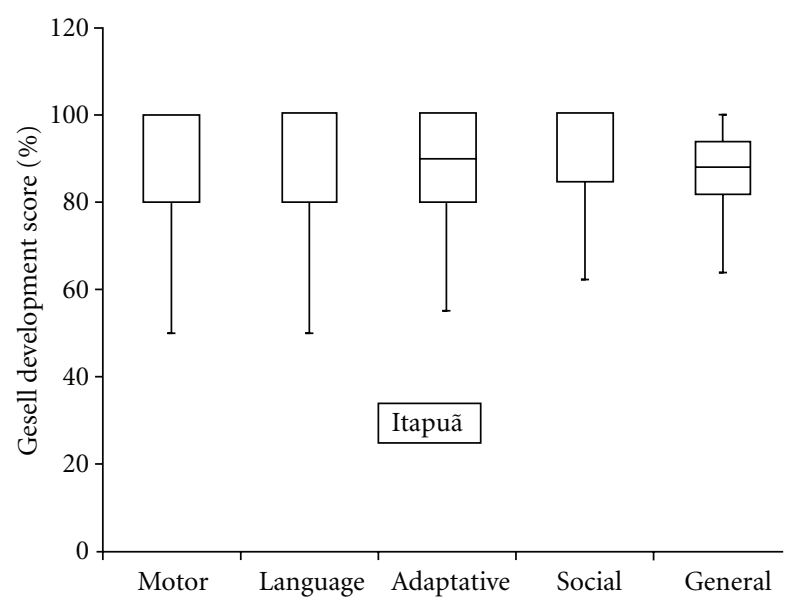

(c)

FIgURE 4: Box plot of Gesell development scores-\% ( $Y$ axis) representing PV, BF, and Itapuã infants.

TABLE 2: Results of the logistic regression of a child showing a score above the median; a negative sign demonstrates a diminished probability.

\begin{tabular}{lcccc}
\hline Parameter & $\begin{array}{c}\text { Coefficient } \\
(\beta)\end{array}$ & $\begin{array}{c}\text { Standard } \\
\text { error }\end{array}$ & Wald $\chi^{2}$ & Pr $>\chi^{2}$ \\
\hline Intercept & -1.5406 & 3.4667 & 0.1975 & 0.6568 \\
Sex & 0.1659 & 0.2940 & 0.3184 & 0.5726 \\
Age & 0.0639 & 0.2216 & 0.0832 & 0.7731 \\
Birth weight & -0.00010 & 0.000286 & 0.1169 & 0.7325 \\
W/H-Z score & 0.2268 & 0.1457 & 2.4229 & 0.1196 \\
W/A-Z score & -0.2695 & 0.2180 & 1.5295 & 0.2162 \\
TCV-EtHg & -0.0157 & 0.00583 & 7.2714 & 0.0070 \\
HHg & -0.0694 & 0.0537 & 1.6691 & 0.1964 \\
Breastfeeding score & -0.00655 & 0.00339 & 3.7265 & 0.0536 \\
\hline
\end{tabular}

W/H: weight for height; W/A: weight for age; VCT-EtHg: thimerosalcontaining vaccine-ethylmercury; HHg: hair mercury; breastfeeding score = age of infant in days/breastfeeding days $\times 100$.

(9.8 ppm-[33]). During breastfeeding, a slow elimination of body $\mathrm{Hg}$ has been linked to continuous exposure of the nursling to $\mathrm{Hg}$ in maternal milk [20]. Indeed, we have also observed that postnatal $\mathrm{HHg}$ increases in breastfed infants [34]. However, despite the increased exposure to EtHg derived from TCV, this source of organic Hg is captured by babies' hair in very small concentrations $[35,36]$; due to the acute nature of TCV exposure, specific EtHg in baby's hair is only correlated with time of last TCV [36]. Therefore, at this early age infants' $\mathrm{HHg}$ showed to be significantly correlated to maternal fish consumption (Figure 2). This is in agreement with previous observations for Amazonian riverine populations [33].

The present study can only suggest association of $\mathrm{Hg}$ exposure from different sources ( $\mathrm{MeHg}$ assessed from $\mathrm{HHg}$ and $\mathrm{EtHg}$ assessed from vaccination cards) and neurobehavioral outcomes. In such observational studies it is difficult to establish a reliable association between markers of exposure and corresponding neurologic effects. While HHg in infants (still breastfeeding and most certainly not eating fish) can be a good indicator of maternal $\mathrm{Hg}$ transferred from fish consumption [33] EtHg could be tracked from vaccination cards. Of the many types of exposure occurring before birth and continuing during the immediate perinatal period, we were able to account for postnatal TCV and breastfeeding. Other aspects related to nutrition and stimulatory factors are complex to tackle in such circumstances. However, the strength of the study is 


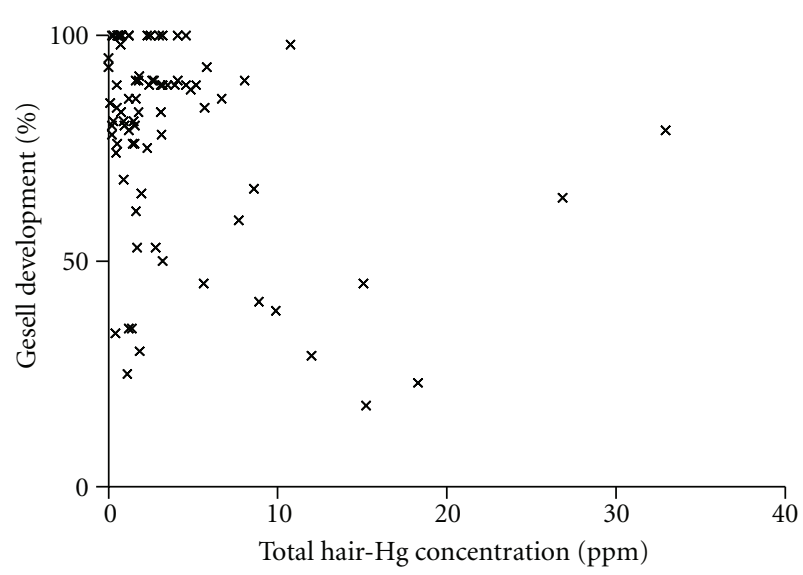

$\times$ Porto Velho

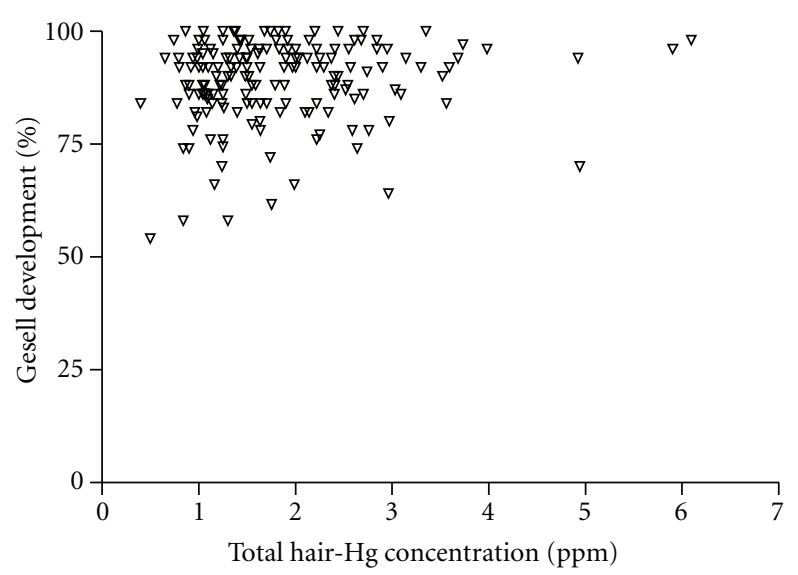

$\nabla$ Bom Futuro

(a)

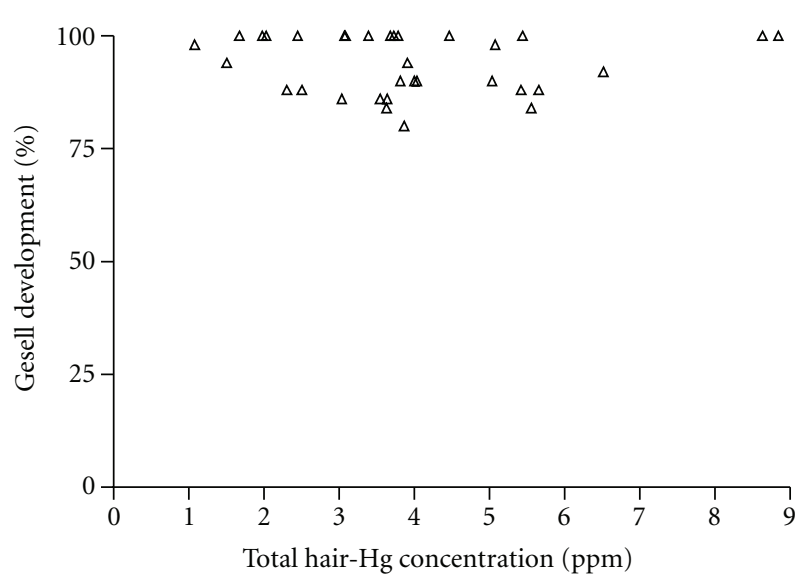

$\Delta$ Itapuã

(c)

FIGURE 5: Scatter plots of total hair-Hg concentrations and Gesell development scores in the 3 settings of tested infants.

that we could compare urban and rural settings accounting indirectly for maternal fish consumption ( $\mathrm{HHg}$ ) and, directly, for exposure to TCV-EtHg. Because Amazonian children are exposed perinatally to TCV-EtHg, neurological assessment at such early ages has to be interpreted with caution. Another weakness relates to the limitation in capturing the many potential confounding variables that can influence experience-dependent synapse formation in such diverse environments (urban and rural settings).

Evidence from animal studies demonstrates the neurodevelopmental impact of prenatal $[37,38]$ and perinatal exposure to vaccine-EtHg $[39,40]$; these studies included infants of several species (mice, rats, and macaques) and suggest that the cumulative effects of TCV-Hg could lead to cognitive and social deficits and delayed acquisition of reflexes. Because this literature is very recent, it has not yet modeled the wide spectrum of human infant diversity and the full interaction with other neurotoxicants [41]. Perinatal TCV-EtHg exposure occurs worldwide, with a wide variation in dose, interval, and postnatal time, in accordance with countries' immunization schedules. Furthermore, nutritional status, degree of poverty, and dispossession attendant on large massed populations in developing countries mean that millions of young children may experience coexposures with other toxic substances that could be more common and more severe.

\section{Conclusions}

For Amazonian infants, not only exposure to environmental fish-MeHg (in maternal diets) but also the additional burden of iatrogenic EtHg (in TCV) is relevant to neurobehavioral testing. In the present case, infants from Porto Velho are exposed to city pollution, Itapuã villagers to more fish$\mathrm{MeHg}$, and Bom Futuro to open-mining operations. However, the neurotoxic effects associated with the combined chemical forms of $\mathrm{Hg}$ seemed sensitive for infants exposed to additional TCV-Hg.

\section{Conflict of Interests}

The authors declare that they have no competing financial interests. 


\section{Acknowledgment}

The present study was partly supported by the National Research Council of Brazil-CNPq (PPG7; CT-HIDRO, project-555516/2006-7; CT-AMAZONIA, Project 575573/ 2008-2).

\section{References}

[1] P. Grandjean and P. Landrigan, "Developmental neurotoxicity of industrial chemicals," The Lancet, vol. 368, no. 9553, pp. 2167-2178, 2006.

[2] P. Grandjean, P. Weihe, R. F. White, and F. Debes, "Cognitive performance of children prenatally exposed to "safe" levels of methylmercury," Environmental Research, vol. 77, no. 2, pp. $165-172,1998$.

[3] K. A. Björnberg, M. Vahter, B. Berglund, B. Niklasson, M. Blennow, and G. Sandborgh-Englund, "Transport of methylmercury and inorganic mercury to the fetus and breastfed infant," Environmental Health Perspectives, vol. 113, no. 10, pp. 1381-1385, 2005.

[4] C. R. Gale, F. J. O’Callaghan, K. M. Godfrey, C. M. Law, and C. N. Martyn, "Critical periods of brain growth and cognitive function in children," Brain, vol. 127, no. 2, pp. 321-329, 2004.

[5] C. R. Gale, F. J. O'Callaghan, M. Bredow, and C. N. Martyn, "The influence of head growth in fetal life, infancy, and childhood on intelligence at the ages of 4 and 8 years," Pediatrics, vol. 118, no. 4, pp. 1486-1492, 2006.

[6] P. W. Stewart, J. Reihman, E. I. Lonky, T. J. Darvill, and J. Pagano, "Cognitive development in preschool children prenatally exposed to PCBs and MeHg," Neurotoxicology and Teratology, vol. 25, no. 1, pp. 11-22, 2003.

[7] A. C. Barbosa, S. R. Silva, and J. G. Dórea, "Concentration of mercury in hair of indigenous mothers and infants from the Amazon basin," Archives of Environmental Contamination and Toxicology, vol. 34, no. 1, pp. 100-105, 1998.

[8] R. C. Marques, J. G. Dórea, C. McManus et al., "Hydroelectric reservoir inundation (Rio Madeira Basin, Amazon) and changes in traditional lifestyle: impact on growth and neurodevelopment of pre-school children," Public Health Nutrition, vol. 14, no. 4, pp. 661-669, 2011.

[9] R. C. Marques, J. G. Dórea, R. S. Leão et al., "The role of methylmercury exposure (from fish consumption) on growth and neuro-development of children under $5 \mathrm{y}$ of age living in a transitioning (tin-mining) area of the Western Amazon, Brazil," Archives of Environmental Contamination and Toxicology, vol. 62, no. 2, pp. 341-350, 2012.

[10] A. Béliveau, M. Lucotte, R. Davidson, L. O. Lopes, and S. Paquet, "Early Hg mobility in cultivated tropical soils one year after slash-and-burn of the primary forest, in the Brazilian Amazon," Science of the Total Environment, vol. 407, no. 15, pp. 4480-4489, 2009.

[11] I. S. Rabitto, W. R. Bastos, R. Almeida et al., "Mercury and DDT exposure risk to fish-eating human populations in Amazon," Environment International, vol. 37, no. 1, pp. 56-65, 2011.

[12] R. C. Marques, J. Garrofe Dórea, W. R. Bastos, M. de Freitas Rebelo, M. de Freitas Fonseca, and O. Malm, "Maternal mercury exposure and neuro-motor development in breastfed infants from Porto Velho (Amazon), Brazil," International Journal of Hygiene and Environmental Health, vol. 210, no. 1, pp. 51-60, 2007.
[13] R. C. Marques, J. G. Dórea, J. V. Bernardi, W. R. Bastos, and O. Malm, "Prenatal and postnatal mercury exposure, breastfeeding and neurodevelopment during the first 5 years," Cognitive and Behavioral Neurology, vol. 22, no. 2, pp. 134$141,2009$.

[14] R. C. Marques, J. G. Dórea, and J. V. E. Bernardi, “Thimerosal exposure (from tetanus-diphtheria vaccine) during pregnancy and neurodevelopment of breastfed infants at 6 months," Acta Paediatrica, vol. 99, no. 6, pp. 934-939, 2010.

[15] J. G. Dórea and R. C. Marques, "Infants' exposure to aluminum from vaccines and breast milk during the first 6 months," Journal of Exposure Science and Environmental Epidemiology, vol. 20, no. 7, pp. 598-601, 2010.

[16] C. J. Passos and D. Mergler, "Human mercury exposure and adverse health effects in the Amazon: a review," Cadernos de Saude Publica, vol. 24, supplement 4, no. 4, pp. S503-S520, 2008.

[17] J. G. Dórea, “Comparing fish-mercury exposed Amazonian children: should not we consider thimerosal-preserved vaccines?" Neuro Toxicology, vol. 30, no. 3, pp. 485-486, 2009.

[18] A. Gesell, A Criança de 0 a 5 Anos, Martins Fontes, São Paulo, Brazil, 6th edition, 2003.

[19] A. Gesell and C.S. Amatruda, Psicologia do Desenvolvimentodo Lactente a Criança Pequena, bases Neuropsicológicas e Comportamentais, Atheneu, São Paulo, Brazil, 1st edition, 2000.

[20] P. Grandjean, P. J. Jørgensen, and P. Weihe, "Human milk as a source of methylmercury exposure in infants," Environmental Health Perspectives, vol. 102, no. 1, pp. 74-77, 1994.

[21] R. C. Marques, J. G. Dórea, J. V. Bernardi, W. R. Bastos, and O. Malm, "Maternal fish consumption in the nutrition transition of the Amazon basin: growth of exclusively breastfed infants during the first 5 years," Annals of Human Biology, vol. 35, no. 4, pp. 363-377, 2008.

[22] J. W. Anderson, B. M. Johnstone, and D. T. Remley, "Breastfeeding and cognitive development: a meta-analysis," American Journal of Clinical Nutrition, vol. 70, no. 4, pp. 525-535, 1999.

[23] J. G. Dórea, "Maternal smoking and infant feeding: breastfeeding is better and safer," Maternal and Child Health Journal, vol. 11, no. 3, pp. 287-291, 2007.

[24] B. E. Lee and E. H. Ha, "Response to commentary "Coexposure and confounders during neurodevelopment: we need them in the bigger picture of secondhand smoke exposure during pregnancy'”, Environmental Research, vol. 112, article 235, 2012.

[25] W. Jedrychowski, "Reply to the correspondence letter by M.D. Majewska: Krakow's children and cognitive function: can the study by Jedrychowski et al. show us the bigger picture?" European Journal of Pediatrics, vol. 171, no. 2, p. 407, 2011.

[26] P. Grandjean, R. F. White, A. Nielsen, D. Cleary, and E. O. Santos, "Methylmercury neurotoxicity in Amazonian children downstream from gold mining," Environmental Health Perspectives, vol. 107, no. 7, pp. 587-591, 1999.

[27] S. Cordier, M. Garel, L. Mandereau et al., "Neurodevelopmental investigations among methylmercury-exposed children in French Guiana," Environmental Research, vol. 89, no. 1, pp. 1$11,2002$.

[28] L. M. Tavares, V. M. Câmara, O. Malm, and E. C. Santos, "Performance on neurological development tests by riverine children with moderate mercury exposure in Amazonia, Brazil," Cadernos de Saúde Pública, vol. 21, no. 4, pp. 11601167, 2005. 
[29] M. F. Fonseca, J. G. Dórea, W. R. Bastos, R. C. Marques, J. P. Torres, and O. Malm, "Poor psychometric scores of children living in isolated riverine and agrarian communities and fishmethylmercury exposure," Neurotoxicology, vol. 29, no. 6, pp. 1008-1015, 2008.

[30] P. Grandjean, P. Weihe, and R. F. White, "Milestone development in infants exposed to methylmercury from human milk," Neurotoxicology, vol. 16, no. 1, pp. 27-33, 1995.

[31] F. Valent, F. Pisa, M. Mariuz et al., "Fetal and perinatal exposure to mercury and selenium: baseline evaluation of a cohort of children in Friuli Venezia Giulia, Italy," Epidemiologia e Prevenzione, vol. 35, no. 1, pp. 33-42, 2011.

[32] S. Mohan, M. Tiller, G. van Der Voet, and H. Kanhai, "Mercury exposure of mothers and newborns in Surinam: a pilot study," Clinical Toxicology, vol. 43, no. 2, pp. 101-104, 2005.

[33] A. C. Barbosa and J. G. Dórea, "Indices of mercury contamination during breast feeding in the Amazon Basin," Environmental Toxicology and Pharmacology, vol. 6, no. 2, pp. 71-79, 1998.

[34] R. C. Marques, J. G. Dórea, W. R. Bastos, and O. Malm, "Changes in children hair-Hg concentrations during the first 5 years: maternal, environmental and iatrogenic modifying factors," Regulatory Toxicology and Pharmacology, vol. 49, no. 1, pp. 17-24, 2007.

[35] J. G. Dórea, W. Wimer, R. C. Marques, and C. Shade, "Automated speciation of mercury in the hair of breastfed infants exposed to ethylmercury from thimerosal-containing vaccines," Biological Trace Element Research, vol. 140, no. 3, pp. 262-271, 2011.

[36] J. G. Dórea, V. L. V. A. Bezerra, V. Fajon, and M. Horvat, "Speciation of methyl- and ethyl-mercury in hair of breastfed infants acutely exposed to thimerosal-containing vaccines," Clinica Chimica Acta, vol. 412, no. 17-18, pp. 1563-1566, 2011.

[37] M. Ida-Eto, A. Oyabu, T. Ohkawara, Y. Tashiro, N. Narita, and M. Narita, "Embryonic exposure to thimerosal, an organomercury compound, causes abnormal early development of serotonergic neurons," Neuroscience Letters, vol. 505, no. 2, pp. 61-64, 2011.

[38] Z. L. Sulkowski, T. Chen, S. Midha, A. M. Zavacki, and E. M. Sajdel-Sulkowska, "Maternal thimerosal exposure results in aberrant cerebellar oxidative stress, thyroid hormone metabolism, and motor behavior in rat pups; sex- and straindependent effects," Cerebellum. In press.

[39] J. G. Dórea, "Integrating experimental (in vitro and in vivo) neurotoxicity studies of low-dose thimerosal relevant to vaccines," Neurochemical Research, vol. 36, no. 6, pp. 927-938, 2011.

[40] M. Duszczyk-Budhathoki, M. Olczak, M. Lehner, and M. D. Majewska, "Administration of thimerosal to infant rats increases overflow of glutamate and aspartate in the prefrontal cortex: protective role of dehydroepiandrosterone sulfate," Neurochemical Research, vol. 37, no. 2, pp. 436-447, 2012.

[41] C. Heilmann, E. Budtz-Jørgensen, F. Nielsen, B. Heinzow, P. Weihe, and P. Grandjean, "Serum concentrations of antibodies against vaccine toxoids in children exposed perinatally to immunotoxicants," Environmental Health Perspectives, vol. 118, no. 10, pp. 1434-1438, 2010. 

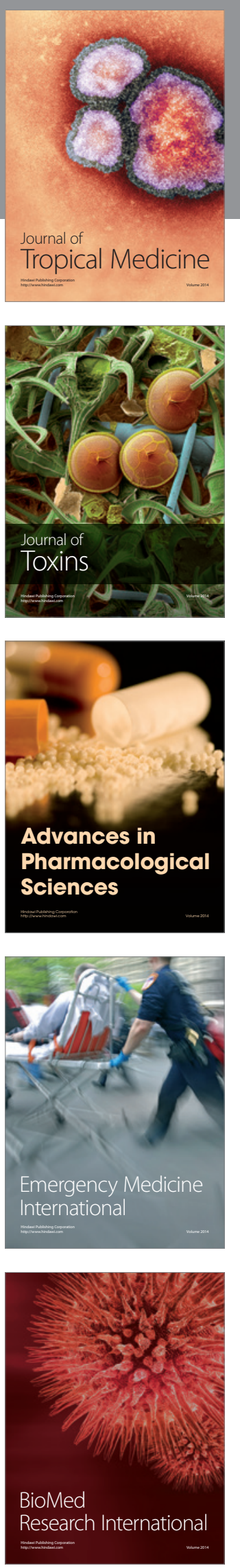
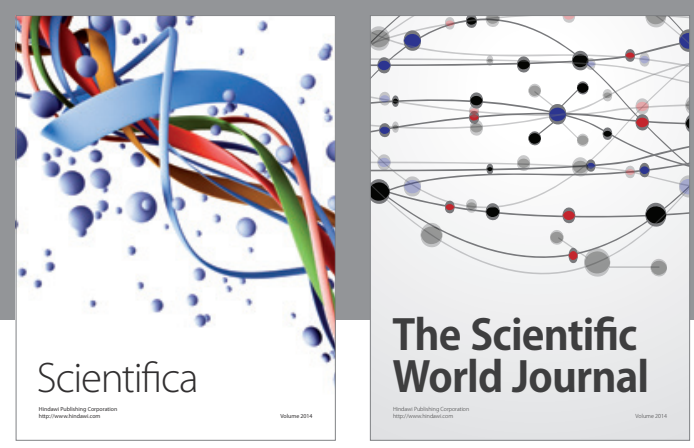

The Scientific World Journal
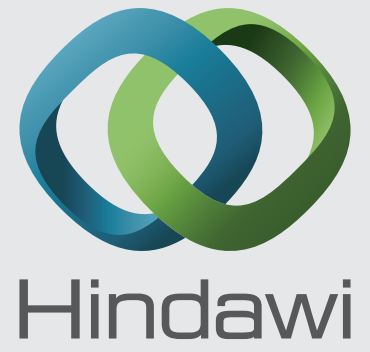

Submit your manuscripts at

http://www.hindawi.com
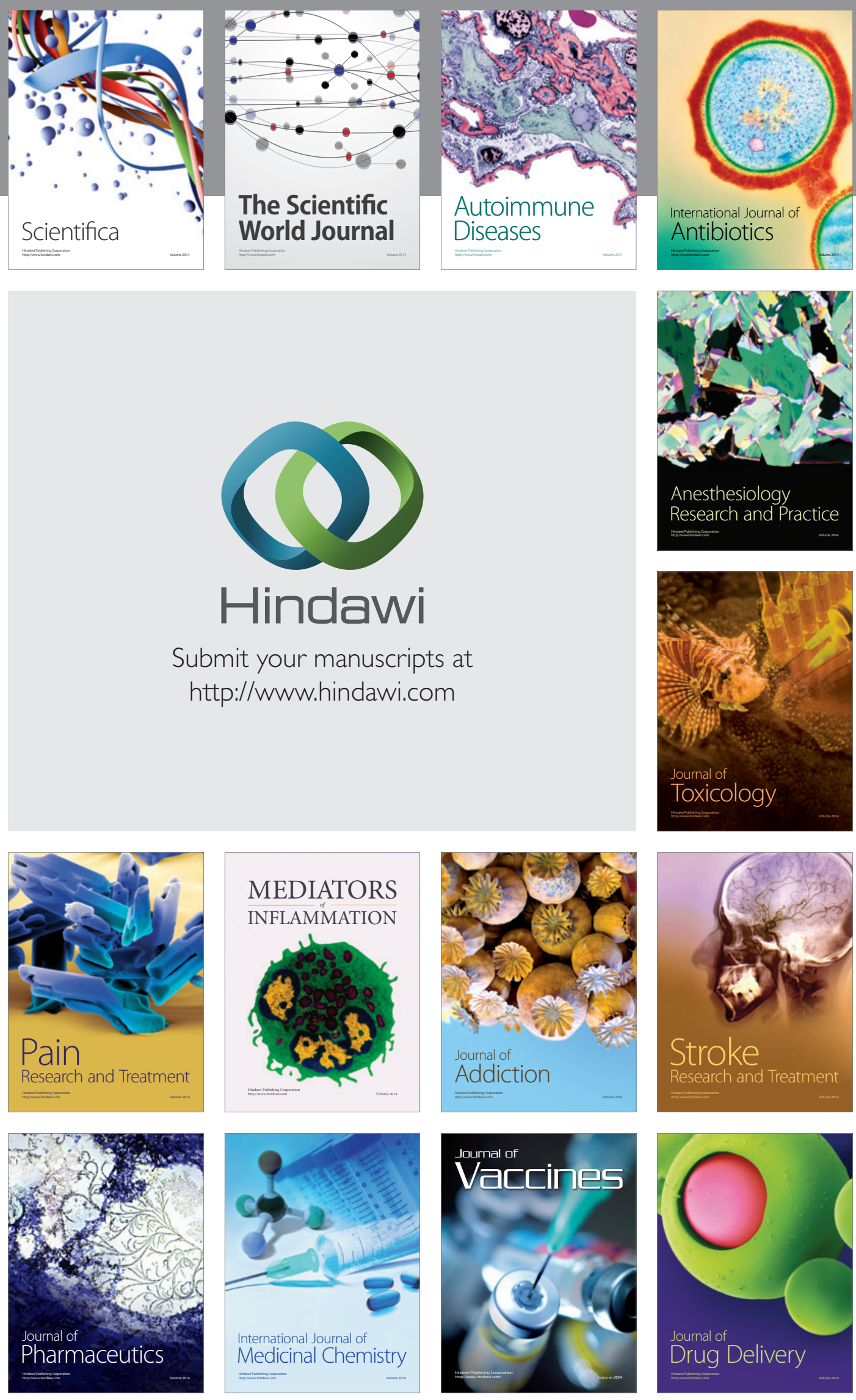